\title{
Laser access and utilization preferences for pediatric ureteroscopy: A survey of the Societies of Pediatric Urology
}

Ray Yong, MD'; Gregory E. Tasian, MD, MSc, MSCE ${ }^{2}$; Kate H. Kraft, MD³; William W. Roberts, $\mathrm{MD}^{3}$; Adam Maxwell, $\mathrm{PhD}^{4}$; Jonathan S. Ellison, $\mathrm{MD}^{1,5}$

${ }^{1}$ Department of Urology, Medical College of Wisconsin, Wauwatosa, WI, United States; ${ }^{2}$ Department of Urology, University of Pennsylvania Perelman School of Medicine; Division of Urology, Children's Hospital of Philadelphia, Philadelphia, PA, United States; ${ }^{3}$ Department of Urology, University of Michigan, Ann Arbor, MI, United States; ${ }^{4}$ Department of Urology, University of Washington, Seattle, WA, United States; ${ }^{5}$ Department of Urology, Medical College of Wisconsin; Division of Pediatric Urology, Children's Hospital of Wisconsin, Milwaukee, WI, United States

Acknowledgements: The authors would like to acknowledge the following individuals for their assistance in this work: Edmond Bedjeti and Shaun Sullivan-Schmidt for survey support and development within SurveyMonkey ${ }^{\mathrm{TM}}$; and Lorraine O'Grady for logistical support via the offices of the SPU.

Cite as: Yong R, Tasian G, Kraft KH, et al. Laser access and utilization preferences for pediatric ureteroscopy: A survey of the societies of pediatric urology. Can Urol Assoc J 2021 October 18; Epub ahead of print. http://dx.doi.org/10.5489/cuaj.7326

Published online October 18, 2021

Corresponding author: Dr. Jonathan S. Ellison, Division of Pediatric Urology, Children's Hospital of Wisconsin, Milwaukee, WI, United States; jellison@chw.org

$* * *$

\section{Abstract}

Introduction: We sought to evaluate laser access and practice variability for pediatric ureteroscopy (URS) across the Societies of Pediatric Urology (SPU) to identify opportunities and barriers for future technology promulgation and evidence dissemination.

Methods: A 25-question survey was sent electronically to members of the SPU. The questionnaire assessed surgeon and hospital characteristics, treatment preferences based on an index case, and information about available laser units. Descriptive and comparative statistical analyses were performed to assess patterns of care and laser accessibility across the SPU.

Results: A total of 105 of 711 (15\%) recipients responded. Seventy-seven respondents (73\%) reported laser ownership, which was associated with greater after-hours laser access (87\% vs. $13 \%, \mathrm{p}<0.01)$. Fifty-eight individuals provided additional laser specifications, of whom $21(36 \%)$ used a high-power laser unit $(>60 \mathrm{~W})$. Standard-power lasers were used more frequently in free- 
standing children's hospitals, as compared to those working within a larger hospital complex ( $75 \%$ vs. $50 \%$, p $=0.049$ ). Variation existed in treatment preferences with respect to dusting (33, $34 \%$ ), fragmentation $(18,19 \%)$, or a hybrid approach (46 respondents, $48 \%$ ). Stone clearance was the most important consideration irrespective of treatment choice.

Conclusions: Variability in surgical preferences and accessibility to laser units exist across pediatric urologists who performing URS. Laser ownership and access to newer technologies vary across practices and may influence treatment options. Understanding access to laser technology will be important when considering opportunities for surgical optimization to improve patient outcomes through future studies.

\section{Introduction}

Up to $30 \%$ of children presenting with an acute episode of upper urinary tract calculi (UUTC) will require surgical intervention. ${ }^{1}$ Over the past decade, ureteroscopy (URS) with laser lithotripsy has become the most common surgical modality for treating children with ureteral and renal calculi. ${ }^{2,3}$ Benefits of URS include the ability to visualize the calculus for directed lithotripsy and active stone extraction with minimal tissue injury from the laser power source. URS has been associated with a lower risk of subsequent retreatments than shock wave lithotripsy (SWL), although at the apparent cost of increased complications. ${ }^{3,4}$ While there are limited data on comparative effectiveness for surgical management of UUTC in the pediatric population, this knowledge gap has been identified as an important area of future study by the National Institute of Diabetes and Digestive and Kidney Diseases (NIDDK). ${ }^{5}$ As such, continued efforts to optimize surgical outcomes for pediatric UUTC must balance the treatment effectiveness against the operative morbidity.

The recent introduction of high powered laser technology into the marketplace has changed treatment paradigms, demonstrating benefits including decreased operative time and avoidance of ureteral access sheaths. ${ }^{6-8}$ Thus access to a more versatile armamentarium of ureteroscopic equipment and energy sources may improve surgical efficiency. ${ }^{9}$ While institutional factors appear to influence choice of surgical intervention (ie. SWL vs URS) in treatment for UUTC in children, availability of laser technologies and technical preferences in the ureteroscopic management for children with UUTC have not been well described. ${ }^{2}$ Comparative effectiveness for surgical management of UUTC in children has been identified as a research priority and several efforts are underway to better refine treatment paradigms in this population. ${ }^{10}$ Specific to these initiatives are efforts to describe modifiable ureteroscopic surgical techniques, such as use of ureteral access sheaths and post-operative ureteral stents as well as the technique of fragmentation (i.e. dusting vs fragment extraction) in the pediatric population. ${ }^{11}$ However, implementing any findings from these efforts will require a broader 
understanding of access to ureteroscopic technologies and procedural variation across the greater pediatric urologic community in order to facilitate incorporation of new technologies. We believe this information would be of interest to several stakeholders who may influence the development, dissemination, and utilization of laser technology including pediatric urologists, industry, hospital administration, and those in health policy.

We surveyed members of the SPU in order to characterize variation in laser lithotripsy access and practices for pediatric URS with an aim to evaluate URS practice patterns and laser accessibility in order to characterize the current state of laser utilization across pediatric urologists and inform future efforts to optimize surgical outcomes for pediatric nephrolithiasis. We hypothesized that laser ownership would be associated with surgical volume, greater afterhours availability, and higher-powered laser devices.

\section{Methods}

\section{Survey development}

A 25-question survey was created by a multidisciplinary research team including 3 pediatric urologists with an interest in pediatric nephrolithiasis, an endourologist treating primarily adult patients, and an experimental physicist expert in laser technology, thus providing content validity; however, the survey instrument lacks construct validation. Survey questions were developed primarily by the senior author (JE) and then reviewed and edited by 4 of the authors (GT, KK, WR, AM) for content and readability. Due to the exploratory nature of the survey and intended audience of pediatric urologists familiar with management of urinary stone disease, we elected to not engage in pilot testing or cognitive interviews for item development. The survey included questions related to surgeon demographics, estimated individual and institutional surgical volumes, accessibility of laser technology, and preferences for treatment strategies including laser settings, lithotripsy technique (dusting vs. fragmentation/extraction), use of pretreatment ureteral stents, and ureteral access sheaths. Additionally, respondents were asked to identify treatment preferences ("dusting" vs "basket extraction and laser fragmentation" vs "hybrid") based on the following index case: "For a $1 \mathrm{~cm}$ renal pelvis stone in a healthy 10 year old child, your preferred ureteroscopic management is..." A full survey can be found in the Supplementary Materials [Supplemental Figure 1]. The survey was created in Survey Monkey ${ }^{\mathrm{TM}}$ (San Mateo, CA).

\section{Survey distribution}

The survey was approved by the SPU and distributed via e-mail to all active, affiliate, corresponding, candidate, and senior members $(n=711)$ in June and July of 2019. Email blast reminders were sent at 2 and 4 weeks. Data were abstracted 6 weeks following initial survey distribution. The SPU list-serve was not directly available to the research team. As such, information regarding the demographics of non-responders cannot be ascertained. Additionally, 
alternative communication strategies for non-responders, beyond the email blasts, were not feasible.

\section{Data definitions}

Ownership of a laser was defined if a respondent answered "own" or "combination of ownership and rental" to the laser ownership survey question. Based on the distribution of the data, surgeon and group volumes were dichotomized into low and high volume categories for both SWL and URS. High volumes were defined as follows: URS group $>50$ cases/year [top quartile]; URS Individual $>30$ cases/year [top quintile]; SWL group $>10$ cases/year [top quintile]. High-power laser was defined as greater than 60 watts. Respondents were asked to identify their practice as either working in a free-standing children's hospital or as children's practice within a larger hospital complex.

\section{Statistical analysis}

Descriptive statistics were utilized to define practice pattern and laser access variability across survey respondents. Chi squared tests were used to assess hospital factors hypothesized to be associated with laser ownership as well as surgeon characteristics hypothesized to be associated with treatment preferences. A P-value less than 0.05 was defined a priori to indicate statistical significance. Statistical analyses were completed in Stata v15.1 (College Station, TX).

\section{Institutional research approval}

Institutional Research Board approval was sought and granted for this project (PRO00033805) prior to proceeding with survey distribution.

\section{Results}

A total of 105 respondents (15\% response rate) from the SPU completed the survey. Data regarding demographics and practice settings are displayed in Table 1. All participants indicated the holmium laser (Ho:YAG) was their preferred energy source. All but one respondent indicated concomitant membership within the AUA and all sections of the AUA were represented.

Seventy-three percent of respondents $(\mathrm{N}=77)$ reported Ho:YAG laser ownership. Neither practice characteristics, case volume, nor laser technology varied by ownership status. However, respondents who owned a laser had greater after-hours (i.e. evenings and weekends) laser access $(87 \%[\mathrm{~N}=66]$ vs $13 \%[\mathrm{~N}=15], \mathrm{P}<0.01)$. Table 2 displays practice characteristics and laser technology stratified by laser ownership. For intra-operative laser support, $50 \%(\mathrm{~N}=$ 52) of respondents depend upon a circulating nurse and $41 \%(\mathrm{~N}=43)$ depend upon an OR technician, with the remainder $(9.5 \%, \mathrm{~N}=10)$ working with other operating room personnel to operate the laser. A sub-analysis was performed to identify current practice variations in respondents who reported access to high power lasers $(\geq 60 \mathrm{~W})$. This sub-analysis found that access to high power lasers was associated with a practice setting within a larger hospital 
complex $(50 \%[\mathrm{~N}=13]$ vs $25 \%[\mathrm{~N}=8], \mathrm{P}=0.04)$, though not associated with academic practice or urolithiasis surgical volumes (either URS or SWL).(data not shown).

Based on an index case of a healthy 10-year-old child with a $1 \mathrm{~cm}$ calculus in the renal pelvis, 33 respondents (34\%) preferred a dusting approach, 18 respondents (19\%) preferred a fragmentation approach, and 46 respondents (48\%) preferred a hybrid approach. An additional 8 respondents chose not to answer. Additional practices stratified by treatment preferences can be seen in Table 3. Respondents who prefer a dusting approach report less frequent access sheath usage ( $p=0.002$ ), while those who prefer a hybrid approach report a greater ability to vary laser pulse settings $(\mathrm{p}=0.015)$. The importance of 7 factors in one's treatment preference is shown in Figure 1. Duration of treatment was chosen more frequently as an influencing factor for those respondents who prefer a hybrid approach $(p=0.038)$. None of the other factors differed significantly across treatment preferences. Stone clearance was unanimously the most influential factor irrespective of treatment preference. Additional variation in practice patterns was assessed which are shown in the Supplemental Table.

\section{Discussion}

As the utilization of URS for children with UUTC increases, understanding practice preferences and access to technology will be essential for widespread efforts to improve outcomes. Variation in treatment modalities (i.e. URS vs SWL) for pediatric UUTC exist across hospital systems, likely driven by local factors and surgeon preference. ${ }^{2,3}$ With greater than 100 respondents representative of a broad complement of practice settings and surgeon experience, we found at majority of respondents use a hospital owned laser. Those urologists indicating laser ownership reported a significantly greater access to after-hours treatment, which may have implications for point-of-care treatment and patient experience. ${ }^{12}$ While neither laser ownership nor surgical volume was associated with use of a high powered laser, surgeons who reported working in free standing children's hospitals had less access to high powered laser technology. Furthermore, just over half of respondents were aware of the laser specifications for their most commonly used device, such as power and variable waveform technology, indicating an opportunity for focused education within the pediatric urology community.

A better understanding of available technology and technical practices could help guide surgical algorithms and capital purchases as high power lasers become more accessible. Our study finds local factors such as laser ownership and practice settings influence access to afterhours laser activity and to newer Ho:YAG laser technology. As such, laser accessibility could thus influence patient outcomes and the patient experience. For instance, after-hours laser use could enable greater point-of-care treatment, thus minimizing need for temporizing ureteral stents and improving patient-reported outcomes, a hypothesis of interest to future study. Meanwhile, access to advanced laser technology may influence treatment decisions and outcomes, as higher powered lasers have been shown to be associated with decreased operative time and avoidance of ureteral access sheaths. ${ }^{6,8,13}$ Few studies exist which support or even 
explore high-powered laser lithotripsy in children, although similar to dissemination of other technological advances in urologic surgery, it follows that use of such lasers could easily gain a foothold in the pediatric realm. Our results support promulgation of such laser technology, especially in health care settings in which adults and children are treated in a shared hospital complex and may represent a positive influence of collaboration across pediatric and adult specialists. Conversely, pediatric urologists who do not share resources with their adult colleagues may not have access high powered lasers. Barriers to adoption of this technology could include absence of high level data in children, costs, or lack of awareness of such technical advances, and should be considered as high powered laser use continues to permeate the pediatric landscape. Additionally, we note that a proportion of our respondents were unaware of the laser power, limiting our assessment of those surgeons with access to high power lasers and highlighting an opportunity for additional surgeon education regarding laser use and functionality.

Advances in laser technology has sparked debate between treatment techniques on dusting versus fragmentation. Varying the settings for laser lithotripsy allows the operator to alter the fragmentation effect, techniques which may be augmented by newer laser technologies. ${ }^{14}$ Matlaga et al have shown no difference in stone clearance or complications when comparing these techniques in adult populations but do acknowledge a paucity of data for children. ${ }^{9}$ Our findings showed a wide variation in treatment preferences exists among engaged pediatric urologists, although treatment efficacy is a key factor for surgeons irrespective of their treatment approach. In accordance with existing literature regarding the benefits of dusting technique and avoidance of ureteral access sheaths, we were not surprised to find our respondents who preferred dusting did so with a goal towards avoidance of access sheaths. ${ }^{9}$ While access sheaths have been safely used in the pediatric population, Wang et al reported a $10 \%$ complication rate and thus, widespread use should be approached with caution, especially in children with a smaller ureteral diameter. ${ }^{15-17}$

This study has several limitations. First, a limited number of potential participants responded to this survey out of the SPU and respondents may represent a select population with an impassioned interest in pediatric endourology. However, those respondents most engaged with treatment of children with nephrolithiasis may be most likely to provide detailed and thorough responses reflective of their current practices. Additionally, the overall number of respondents is similar to recently published surveys from the same society. ${ }^{18-20}$ As senior, corresponding, and affiliate members, who may or may not be actively practicing, were included in the e-mail blast, this could have artificially decreased the apparent response rate from active and practicing pediatric urologists. Survey responses did not categorize membership status and could not further ascertain the membership type. Second, as the responses obtained may have been influenced by those more dedicated to pediatric stone disease, our survey may overestimate the degree of laser access. Additionally, respondents could cluster within institutions. The de- 
identified nature of the survey process precludes a more detailed assessment of this potential clustering. However, no more than $23 \%$ of respondents were from each AUA section, suggesting a broad regional representation. Lastly, the ever-changing landscape of endourology could render these results out of date in just a few years. For that reason, continued engagement in this arena will be essential. Lastly, reliance on electronic survey distribution, while convenient, limits the survey to those with computer access and limits the ability of the respondents to ask clarifying questions of the research team.

In spite of our limitations, our study has several important implications for future endoscopic management of pediatric stone disease, which are especially notable given the predominant preference towards URS for surgical management of UUTC in children. First, institutional factors appear to influence laser access, both in terms of treatment availability and access to high powered equipment. Both factors could be hypothesized to influence patient outcomes. As additional data further inform ideal treatment patterns, understanding barriers to laser access will be essential for planning knowledge dissemination and implementation across our specialty. Second, embedded technical variability exists across surgeons. Many, if not all, of these technical preferences lack supporting data, and these findings support efforts targeted to harness surgical variability in order to define optimal treatment parameters. Currently the Pediatric KIDney Stone Care Improvement Network (PKIDS) has an ongoing multi-institutional prospective cohort study for children undergoing surgical treatment for stone disease, evaluating factors that influence treatment outcomes and patient experience (clinicaltrials.gov

NCT04285658). ${ }^{10}$ Our findings will be key preliminary data to understand optimal targets for and feasibility of evidence dissemination to engaged pediatric urologists across the SPU.

\section{Conclusions}

Our data show Ho:YAG laser access and availability to be influenced by practice characteristics, with respondents from free-standing children's hospitals reporting less use of high powered laser technology, and those with laser ownership reporting greater after-hours laser access. Many respondents were not aware of laser power or accessory settings, representing an opportunity for focused education in this realm. We identified a wide variability in pediatric ureteroscopic practice patterns and treatment preferences, which has the potential to be harnessed within broad collaborative studies to identify optimal operative strategies for pediatric stone disease. 


\section{References}

1. Ellison JS, Merguerian PA, Fu BC, et al. Use of medical expulsive therapy in children: An assessment of nationwide practice patterns and outcomes. J Pediatr Urol 2017; 13: 509 e501-509 e507. 2017/05/10. DOI: 10.1016/j.jpurol.2017.03.022.

2. Wang HH, Huang L, Routh JC, et al. Shock wave lithotripsy vs ureteroscopy: variation in surgical management of kidney stones at freestanding children's hospitals. J Urol 2012; 187: 1402-1407. 2012/02/22. DOI: 10.1016/j.juro.2011.12.010.

3. Ellison JS, Shnorhavorian M, Oron A, et al. Risk factors for repeat surgical intervention in pediatric nephrolithiasis: A Pediatric Health Information System database study. $J$ Pediatr Urol 2018; 14: 245 e241-245 e246. 2018/03/28. DOI: 10.1016/j.jpurol.2018.02.017.

4. Tejwani R, Wang HH, Wolf S, et al. Outcomes of Shock Wave Lithotripsy and Ureteroscopy for Treatment of Pediatric Urolithiasis. J Urol 2016; 196: 196-201. 2016/03/22. DOI: 10.1016/j.juro.2016.02.2975.

5. Schwaderer A and Tasian GE. Pediatric Nephrolithiasis. In: Urinary Stone Disease: Research Challenges and Opportunities Bethesda, MD, 2015, National Institute of Diabetes and Digestive and Kidney Diseases.

6. Santiago JE, Hollander AB, Soni SD, et al. To dust or not to dust: a systematic review of ureteroscopic laser lithotripsy techniques. Current urology reports 2017; 18: 32.

7. Aldoukhi A and Ghani K. Ureteroscopic holmium laser lithotripsy using the Moses technology. European Urology Supplements 2018; 17: e1918.

8. Hardy LA, Gonzalez DA, Irby PB, et al. Fragmentation and dusting of large kidney stones using compact, air-cooled, high peak power, 1940-nm, thulium fiber laser. In: Therapeutics and Diagnostics in Urology 2018 2018, p.104680O. International Society for Optics and Photonics.

9. Matlaga BR, Chew B, Eisner B, et al. Ureteroscopic Laser Lithotripsy: A Review of Dusting vs Fragmentation with Extraction. J Endourol 2018; 32: 1-6. 2017/10/25. DOI: 10.1089/end.2017.0641.

10. ClinicalTrials.gov. Pediatric KIDney Stone (PKIDS) Care Improvement Network (PKIDS), https://clinicaltrials.gov/ct2/show/NCT04285658 (2020).

11. Tasian GE and Ellison JS. The Surgical Improvement Cycle: Improving Surgical Outcomes through Partnerships and Rigor. J Urology 2021.

12. He Q, Xiao KW, Chen YT, et al. Which is the best treatment of pediatric upper urinary tract stones among extracorporeal shockwave lithotripsy, percutaneous nephrolithotomy and retrograde intrarenal surgery: a systematic review. Bmc Urology 2019; 19: 98. DOI: ARTN 98

10.1186/s12894-019-0520-2.

13. Aldoukhi AH, Roberts WW, Hall TL, et al. Understanding the Popcorn Effect During Holmium Laser Lithotripsy for Dusting. Urology 2018; 122: 52-57. 2018/09/09. DOI: 10.1016/j.urology.2018.08.031.

14. Faure A and Boissier R. Optimal settings for the Holmium: YAG laser in pediatric endourology: Tips and tricks. J Pediatr Urol 2020; 16: 244-250. 2020/04/01. DOI: 10.1016/j.jpurol.2020.03.002. 
15. Marchetti KA, Lee T, Raja N, et al. Extracorporeal shock wave lithotripsy versus ureteroscopy for management of pediatric nephrolithiasis in upper urinary tract stones: multi-institutional outcomes of efficacy and morbidity. J Pediatr Urol 2019; 15: 516 e511-516 e518. 2019/07/22. DOI: 10.1016/j.jpurol.2019.06.006.

16. Wang HH, Huang L, Routh JC, et al. Use of the ureteral access sheath during ureteroscopy in children. J Urol 2011; 186: 1728-1733. 2011/08/23. DOI: 10.1016/j.juro.2011.03.072.

17. Anbarasan R, Griffin SJ and Somani BK. Outcomes and Long-Term Follow-Up with the Use of Ureteral Access Sheath for Pediatric Ureteroscopy and Stone Treatment: Results from a Tertiary Endourology Center. J Endourol 2019; 33: 79-83. 2018/12/05. DOI: 10.1089/end.2018.0448.

18. Ahn JJ, Ellison JS and Merguerian PA. A Societies for Pediatric Urology survey of opioid prescribing practices after ambulatory pediatric urology procedures. J Pediatr Urol 2019; 15: 451-456. 2019/06/05. DOI: 10.1016/j.jpurol.2019.04.025.

19. Rehfuss A, Bogaert G and Kogan B. Spinal anesthesia in children: most pediatric urologists are not on board. Journal of Pediatric Urology 2019; 15: 263. e261-263. e265.

20. Mistry PK, Morganstern BA, Ahmed H, et al. Attitudes towards child life specialists and their utilization within pediatric urology. Canadian Journal of Urology 2019; 26: 10022-10025.

Competing interests: Dr. Tasian serves on the scientific advisory boards for Allena Pharmaceuticals, Novome Biotechnology, and Dicerna Pharmaceuticals; and is a consultant for Alnylam. Dr. Roberts is a consultant for Boston Scientific. Dr. Maxwell has equity and consulting arrangements with SonoMotion, Inc and is a consultant for Boston Scientific. Dr. Ellison is a consultant for Dicerna Pharmaceuticals. The remaining author report no personal or financial conflicts related to this work. 


\section{Figures and Tables}

Fig. 1. Factors influencing surgeon preference for dusting, fragmentation, or hybrid approaches to laser lithotripsy. The total number of respondents for each factor is noted below the $\mathrm{x}$-axis.

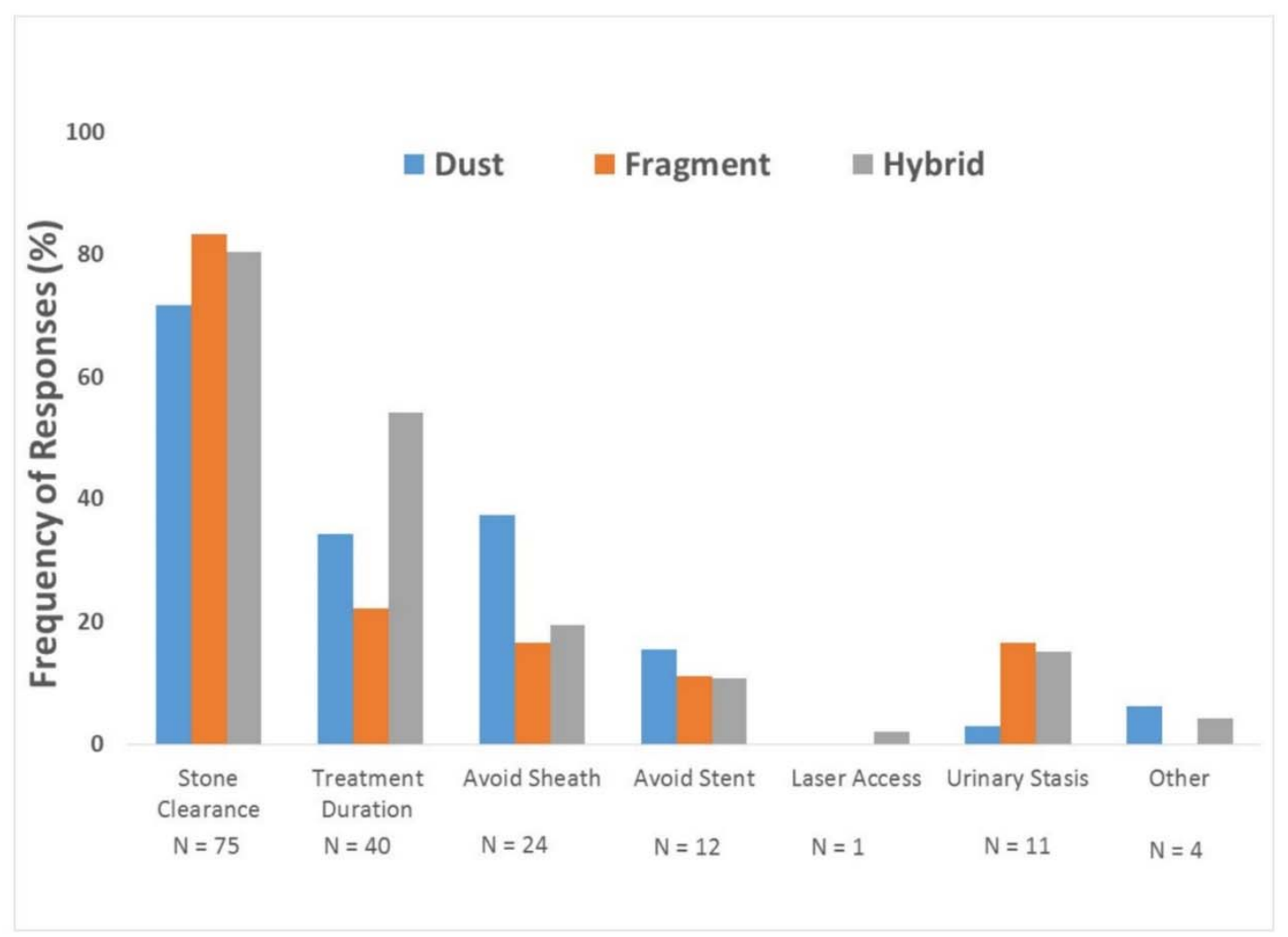




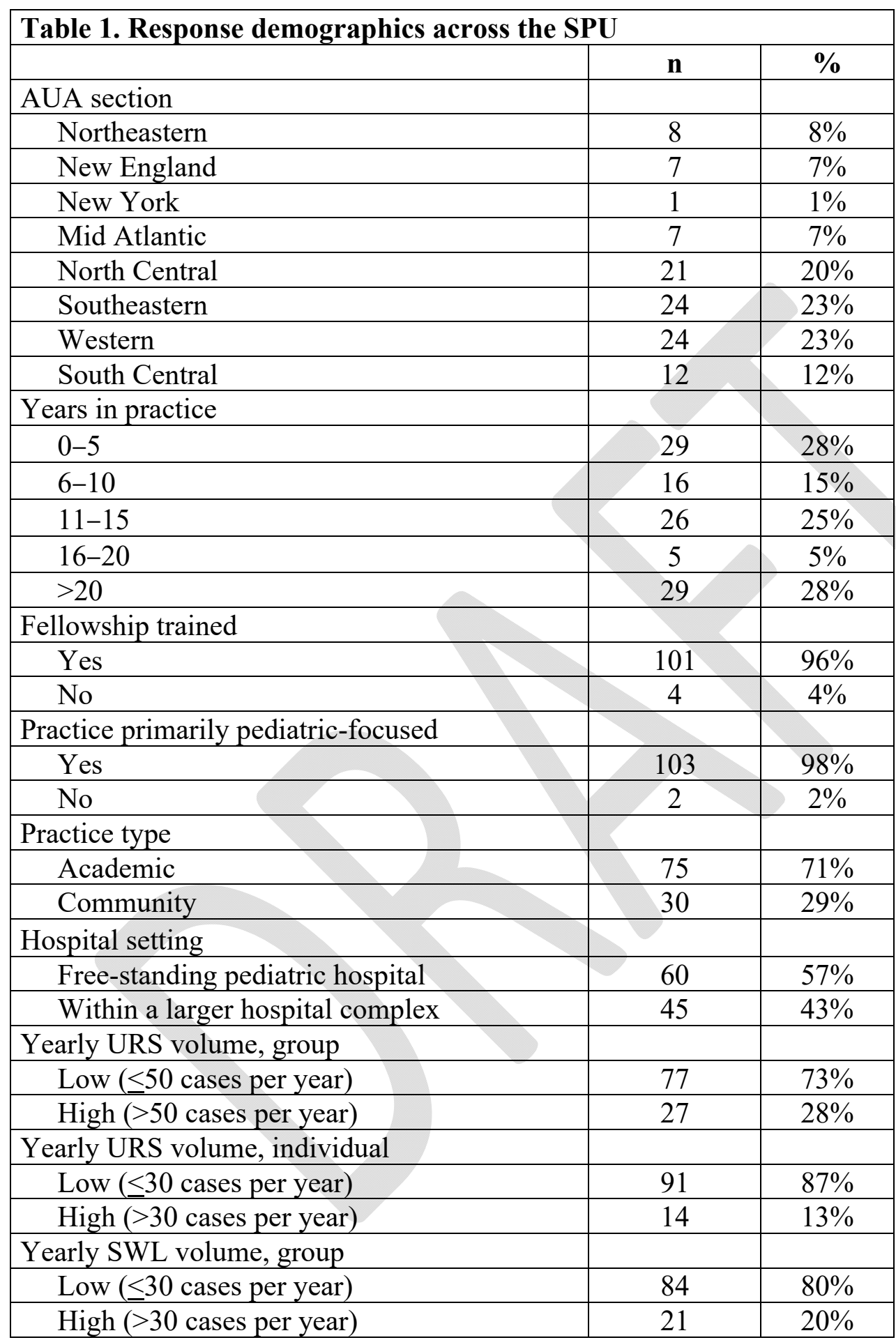

AUA: American Urological Association; SPU: Societies of Pediatric Urology; SWL: shockwave lithotripsy; URS: ureteroscopy. 


\begin{tabular}{|c|c|c|c|}
\hline & Own & Rent & $\mathbf{p}$ \\
\hline \multicolumn{4}{|l|}{ Practice type } \\
\hline Academic & $57(77 \%)$ & $17(23 \%)$ & 0.34 \\
\hline Community & $20(67 \%)$ & $10(33 \%)$ & \\
\hline \multicolumn{4}{|l|}{ Hospital setting } \\
\hline $\begin{array}{l}\text { Free-standing children's } \\
\text { hospital }\end{array}$ & $42(71 \%)$ & $17(29 \%)$ & 0.5 \\
\hline $\begin{array}{l}\text { Within a larger hospital } \\
\text { complex }\end{array}$ & $35(78 \%)$ & $10(22 \%)$ & \\
\hline \multicolumn{4}{|l|}{ Group URS volume } \\
\hline Low ( $<50$ cases per year) & $55(71 \%)$ & $22(29 \%)$ & 0.44 \\
\hline High ( $>50$ cases per year) & $22(81 \%)$ & $5(19 \%)$ & \\
\hline \multicolumn{4}{|l|}{ Group SWL volume } \\
\hline Low $(<30$ cases per year $)$ & $62(75 \%)$ & $21(25 \%)$ & 0.43 \\
\hline High ( $>30$ cases per year) & $15(71 \%)$ & $6(29 \%)$ & +2 \\
\hline \multicolumn{4}{|l|}{ After-hours laser access } \\
\hline Yes & $66(87 \%)$ & $10(13 \%)$ & $<0.01$ \\
\hline No & $11(39 \%)$ & $17(61 \%)$ & \\
\hline \multicolumn{4}{|l|}{ Laser power } \\
\hline Standard $(<60 \mathrm{~W})$ & $30(81 \%)$ & $7(19 \%)$ & 0.25 \\
\hline $\operatorname{High}(>60 \mathrm{~W})$ & $17(81 \%)$ & $4(19 \%)$ & \\
\hline Unsure & $30(67 \%)$ & $15(33 \%)$ & \\
\hline \multicolumn{4}{|l|}{ Pulse settings } \\
\hline Variable & $41(73 \%)$ & $15(27 \%)$ & 0.92 \\
\hline Non-variable & $13(72 \%)$ & $5(28 \%)$ & \\
\hline Unsure & $23(77 \%)$ & $7(23 \%)$ & \\
\hline
\end{tabular}

SWL: shockwave lithotripsy; URS: ureteroscopy. 


\begin{tabular}{|l|c|c|c|c|c|}
\hline \multicolumn{7}{|l|}{ Table 3. Factors associated with treatment preferences } \\
\hline & Total & Dusting & Fragmentation & Hybrid & p \\
\hline Demographics & & & & & \\
\hline Years in practice & & & & & \\
\hline$\leq 10$ & 44 & $10(23 \%)$ & $10(23 \%)$ & $24(55 \%)$ & 0.124 \\
\hline$>10$ & 52 & $22(42 \%)$ & $8(15 \%)$ & $22(42 \%)$ & \\
\hline Individual URS volume & & & & & \\
\hline $\begin{array}{l}\text { Low }(<30 \text { cases per } \\
\text { year) }\end{array}$ & 82 & $30(37 \%)$ & $13(16 \%)$ & $39(48 \%)$ & 0.116 \\
\hline $\begin{array}{l}\text { High }(>30 \text { cases per } \\
\text { year) }\end{array}$ & 14 & $2(14 \%)$ & $5(36 \%)$ & $7(50 \%)$ & \\
\hline Practice patterns & & & & & \\
\hline Pre-stenting & & & & & \\
\hline$<60 \%$ of cases & 44 & $17(39 \%)$ & $8(18 \%)$ & $19(43 \%)$ & 0.583 \\
\hline$>60 \%$ of cases & 52 & $15(29 \%)$ & $10(19 \%)$ & $27(52 \%)$ & \\
\hline Access sheath use & & & & & \\
\hline$<50 \%$ & 64 & $26(41 \%)$ & $6(9 \%)$ & $32(50 \%)$ & $\mathbf{0 . 0 0 2}$ \\
\hline$>50 \%$ & 32 & $6(19 \%)$ & $12(38 \%)$ & $14(44 \%)$ & \\
\hline Laser access & & & & & \\
\hline Laser ownership & & & & & \\
\hline Own & 74 & $24(32 \%)$ & $12(16 \%)$ & $38(51 \%)$ & 0.372 \\
\hline Rent & 22 & $8(36 \%)$ & $6(27 \%)$ & $8(36 \%)$ & \\
\hline Laser power & & & & & \\
\hline Low power $(<60 \mathrm{~Hz})$ & 36 & $15(42 \%)$ & $5(14 \%)$ & $16(44 \%)$ & 0.251 \\
\hline High power $(\geq 60 \mathrm{~Hz})$ & 19 & $5(26 \%)$ & $6(32 \%)$ & $8(42 \%)$ & \\
\hline Pulse setting variation & & $15(31 \%)$ & $6(12 \%)$ & $28(57 \%)$ & $\mathbf{0 . 0 1 5}$ \\
\hline Yes & 49 & $7(39 \%)$ & $7(39 \%)$ & $4(22 \%)$ & \\
\hline No & 18 & & & \\
\hline
\end{tabular}

URS: ureteroscopy. 